\title{
Gaussian Mixture Model for MRI Image Segmentation to Build a Three-Dimensional Image on Brain Tumor Area
}

\author{
1,2 Anindya Apriliyanti Pravitasari*, ${ }^{2}$ Nur Iriawan, ${ }^{2}$ Siti Azizah Nurul Solichah, \\ ${ }^{2}$ Irhamah, ${ }^{2}$ Kartika Fithriasari, ${ }^{2}$ Santi Wulan Purnami and \\ ${ }^{3}$ Widiana Ferriastuti \\ ${ }^{1}$ Department of Statistics, Faculty of Mathematics and Natural Sciences, Universitas Padjadjaran \\ 45363 Bandung, Indonesia \\ ${ }^{2}$ Department of Statistics, Faculty of Science and Data Analytics \\ Institut Teknologi Sepuluh Nopember, 60111 Surabaya, Indonesia \\ ${ }^{3}$ Department of Radiology, Faculty of Medicine, Universitas Airlangga \\ 60115 Surabaya, Indonesia \\ *Corresponding author: anindya.apriliyanti@unpad.ac.id
}

Article history

Received: 20 June 2019

Received in revised form: 19 August 2020

Accepted: 5 October 2020

Published on line: 1 December 2020

\begin{abstract}
A brain tumor is one of the deadly diseases that attack the central and nervous system. The treatment of brain tumor, need high accuracy and precision. Brain tumor detection through Magnetic Resonance Imaging (MRI) has two-dimensional output with three perspectives, namely sagittal, coronal, and axial. These different perspectives need to be seen one by one to determine the location and size of the tumor. To solve the problem, this study constructs the three-dimensional visualization perspective of MRI images. The tumor area in MRI image is segmented as a region of interest (ROI) by employing the Gaussian Mixture Model (GMM) with Expectation-Maximization as the optimization technique. These couple segmentation methods have revealed significant gain as a clear boundary of the tumor area to separate from the healthy part of the brain and an estimated tumor volume from sagittal, coronal, and axial perspectives. Furthermore, these findings have been successfully visualized in 3D construction of the tumor position on the left side of the patient's head with an estimated volume of $749 \mathrm{~mm}^{3}$.
\end{abstract}

Keywords brain tumor segmentation; Expectation-Maximization; Gaussian Mixture Model; three-dimensional image.

Mathematics Subject Classification 20E28, 62E99, 33F99, 68U10.

\section{Introduction}

The brain is an essential human organ with the ability to control the whole part of the body. An abnormal condition of a brain is a condition associated with neurological disorders, one of such diseases is a brain tumor that grows inside the central spinal canal. A brain tumor is a deadly disease since it has a high mortality rate. According to WHO, in 2018, Indonesia has 
5,323 brain and nervous system tumors incidence with 4,229 cases of mortality [1]. This is why the brain tumor is considered to be an important topic. The detection of a brain tumor can be done through medical devices called Magnetic Resonance Imaging (MRI). Unlike Computed Tomography (CT) which requires ionizing radiation, MRI is based on the interaction between radio waves and the nucleus of a hydrogen atom in the body by the presence of a magnetic field [2]. A higher MRI Tesla power delivers a better resolution and is very useful for producing clear image visualization. General Hospital Dr. Soetomo provides radiological services including MRI 3 and 1.5 Tesla. However, the latter as the lower Tesla power of MRI is favorable since its more affordable and covered by the Indonesian governments social security (BPJS). This study aims to enhance the image quality of 1.5 Tesla MRI using the image segmentation technique.

Previous research had been developed for image segmentation. Clustering analysis is one of the unsupervised learning for segmentation methodology. The unsupervised methodology is used in this study due to the limited number of data. Several cluster analyses for image segmentation are provided by Martínez-Usó et al. [3] that use the Hierarchical Clustering selection process in image segmentation, and Pestunov et al. [4] that employs the Hierarchical Clustering algorithm for Multispectral Images. Partitioning algorithm technique also widely used in image segmentation, such as K-means Cluster for color-based image segmentation by Rohit et al. [5]. Moreover, Muruganadham et al. [6] also used the K-means and K-medoid to identify the diseases in a leaf's images. Fuzzy based clustering such as FCM that employed to segmenting the MRI brain image is studied by Huang et al. [7]. Lee et al. [8] also did a similar study but used the Population Diameter Independent algorithm as the method of segmentation. The hierarchical clustering or partitioning algorithm is favorable since it's easy to use [9], however, it has a disability to recognize the pattern of MRI image [10]. Modelbased clustering is the most appropriate tool to overcome this problem, especially when facing a limited number of data [11]. The Gaussian Mixture Model (GMM) is one of model-based clustering that will be employed to the MRI data image with Expectation-Maximization as the optimization technique. The research on image segmentation, especially in clinical data, often uses GMM rather than other distributions [12]. The cluster validation is done by calculating the Silhouette Coefficient $(S C)$ to provide the optimum number of clusters.

The segmentations are done to the whole MRI slices in three perspective images of sagittal, coronal, and axial. The slices of MRI images are two-dimensional images that are set as a collection of the sequential images in each perspective. To make a medical decision related to the location and volume of the tumor, the radiologist have to inspect every slice in each perspective. The three perspective segmented images will be arranged together in a compact manner to form a three-dimensional (3D) structure using volume viewer via Matlab. Using the 3D model, the volume of a brain tumor can easily be known and calculate under the ellipsoidal formula. This study is designed to help radiologists determine the brain tumor volume and location more effectively and efficiently.

\section{Methodology}

The methodology is briefed under two important works, the first is segmenting image using GMM and the second is constructing the 3D model using the volume viewer in Matlab. The two assignments are carried out in series, where the results of three perspective segmented images using GMM are becomes the input to build the 3D models. All procedures could be visualized in Figure 1, while the explanation of several methodologies is providing in the next 
sub-sections.

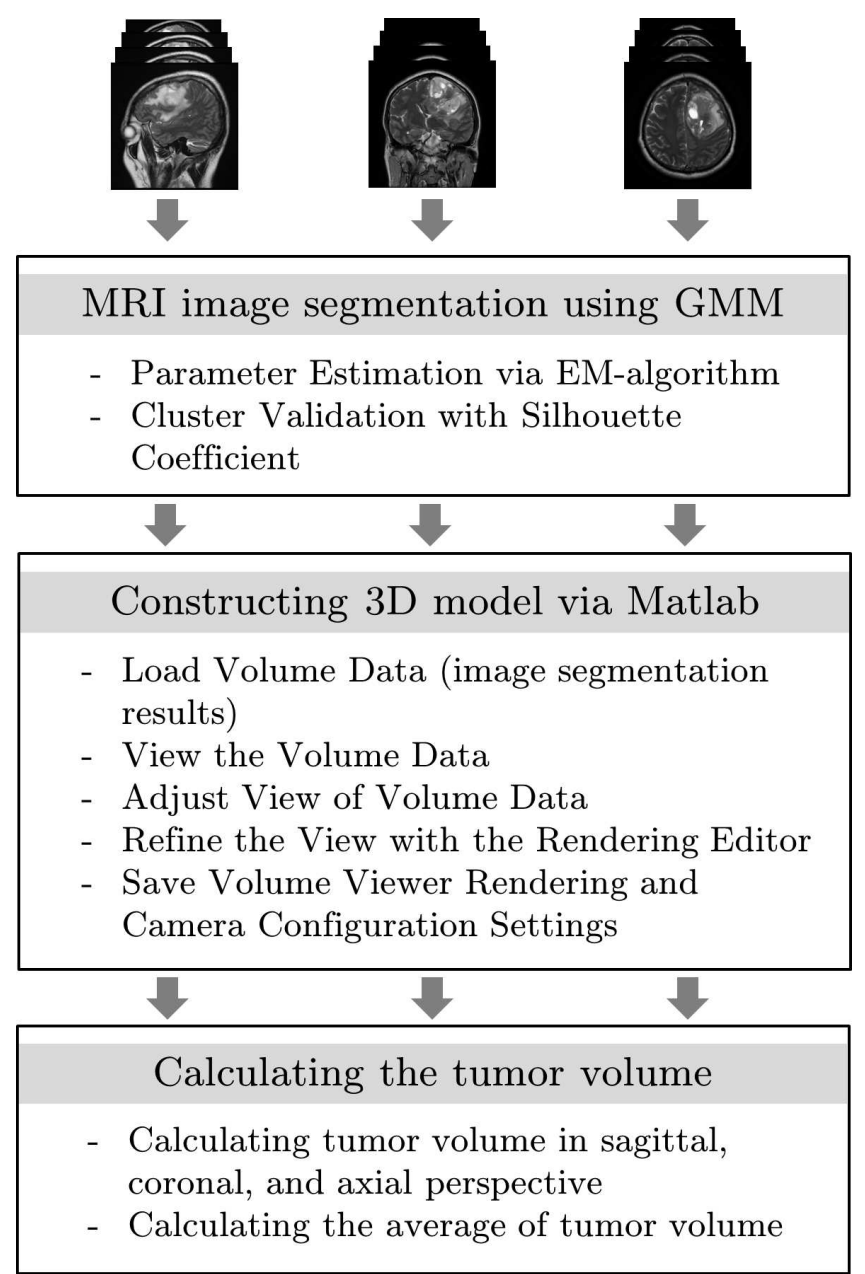

Figure 1: Data Analysis Procedure

\subsection{Image Segmentation using Gaussian Mixture Model (GMM)}

The first task is segmenting the MRI image by employing the GMM. This task includes the optimization using the EM algorithm and cluster validation using Silhouette Coefficient. Suppose we have a grayscale MRI image in size $256 \times 256$ pixels. Each pixel will have a grayscale intensity between 0 and 255. The lower the intensity, the darker the color, and vice versa. The pixel in MRI image will be named as variable $x_{i}, i=1,2, \ldots N$, which $N$ is number of pixels. Each pixel will be grouped into $K$ clusters, which every cluster is assumed to have the Gaussian distribution. For general, the GMM is the compilation of some Gaussian distributions whose form a new following distribution [13]:

$$
f\left(x_{i} \mid \mathbf{p}, \boldsymbol{\mu}, \boldsymbol{\sigma}\right)=\sum_{j=1}^{K} p_{j} f\left(x_{i} \mid \mu_{j}, \sigma_{j}^{2}\right),
$$


where $f\left(x_{i} \mid \mathbf{p}, \boldsymbol{\mu}, \boldsymbol{\sigma}\right)$ is the probability density function (p.d.f) of the mixture model, $f\left(x_{i} \mid \mu_{j}, \sigma_{j}^{2}\right)$ is the Gaussian p.d.f of the $j^{\text {th }}$ components of the mixture model, as follows:

$$
f\left(x_{i} \mid \mu_{j}, \sigma_{j}^{2}\right)=\frac{1}{\sqrt{2 \pi} \sigma_{j}} \exp \left(-\frac{1}{2}\left(\frac{x_{i}-\mu_{j}}{\sigma_{j}}\right)^{2}\right),
$$

with $\mu_{j}$ and $\sigma_{j}$ are parameters for Gaussian p.d.f, $\mathbf{p}$ is vector parameter proportions which has elements $\left(p_{1}, p_{2}, \ldots, p_{K}\right)$, that satisfy $0 \leq p \leq 1$ and $\sum_{j=1}^{K} p_{j}=1$, and $K$ is the number of distribution components in the mixture model.

The mixture model has been used in various disciplines, some of which are Biology, Physics, Education, and Economics. For the Medical discipline, it is based on the fact that patterns of grayscale data inform a multimodal that can be expressed as a mixture of several distributions whose parameters are known. For example, it contains some Gaussian with the Expectation Maximization (EM) Algorithm as the optimization of parameters estimation.

\subsection{Parameter Estimation using EM Algorithm}

The parameter estimation is conducted using the EM algorithm. This algorithm is often used as an iterative computational approach to the Newton-Raphson method or other more complicated techniques in estimating parameters through Maximum Likelihood Estimation (MLE) [14]. The following is the EM Algorithm for estimating the GMM.

\section{Algorithm 1. The EM Algorithm for esGMM}

1. Initialization parameters $\left\{\mu_{j}^{(0)}, \sigma_{j}^{2(0)}, p_{j}^{(0)}\right\}$ and set $t=0$.

2. E-Step: Calculate $z_{i j}, i=1,2, \ldots, N$ and $j=1,2, \ldots, K$ the latent variable which indicates the label of a cluster.

$$
z_{i j}^{(t+1)}=\frac{p_{j}^{(t)} f\left(x_{i} \mid \mu_{j}^{(t)}, \sigma_{j}^{(t)}\right)}{\sum_{k=1}^{K} p_{k}^{(t)} f\left(x_{i} \mid \mu_{k}^{(t)}, \sigma_{k}^{(t)}\right)} .
$$

3. M-Step: Re-estimate parameters $\left\{\mu_{j}, \sigma_{j}^{2}, p_{j}\right\}, j=1,2, \ldots, K$ with the recursion formulae

$$
\begin{gathered}
\mu_{j}^{(t+1)}=\frac{\sum_{i=1}^{N} z_{i j}^{(t+1)} x_{i}}{\sum_{i=1}^{N} z_{i j}^{(t+1)} .} \\
\left(\sigma_{j}^{2}\right)^{(t+1)}=\frac{\sum_{i=1}^{N} z_{i j}^{(t+1)}\left(x_{i}-\mu_{j}^{(t+1)}\right)^{2}}{\sum_{i=1}^{N} z_{i j}^{(t+1)}} . \\
p_{j}^{(t+1)}=\frac{1}{N} \sum_{i=1}^{N} z_{i j}^{(t+1)} .
\end{gathered}
$$


4. Evaluate the parameter values, if the difference of parameter values in iteration $t$ and $(t-1)$ fulfilled the criteria of convergence $(<\varepsilon)$, then iteration stop, otherwise return to step 2 .

\subsection{Cluster Validation}

Cluster validation is needed to provide the optimum number of clusters. In this study, Silhouette Coefficient $(S C)$ is used to calculate cohesion and separation. Here are the steps to calculate $S C[15]$.

Algorithm 2. Silhouette Coefficient

1) Calculate $a_{i}^{j}$ as the average square Euclidean distance to $i^{t h}$ data with other data in a single cluster.

2) Calculate $b_{i}^{j}$ as the minimum of average square Euclidean distance of $i^{\text {th }}$ data to other data from the different clusters.

3) Calculate the $S C$ value of every $i^{\text {th }}$ data at $j^{\text {th }}$ cluster using equation (7)

$$
S C_{i}^{j}=\frac{b_{i}^{j}-a_{i}^{j}}{\max \left(a_{i}^{j}, b_{i}^{j}\right)} .
$$

4) Calculate $S C$ for each $j^{\text {th }}$ cluster using equation (8), where $n_{j}$ is the number of pixels in $j^{\text {th }}$ cluster.

$$
S C_{j}=\frac{1}{n_{j}} \sum_{i=1}^{n_{j}} S C_{i}^{j} .
$$

5) Calculate the overall $S C$ with the following equation

$$
S C=\frac{1}{K} \sum_{j=1}^{K} S C_{j} .
$$

$S C$ value is between -1 and 1 . A value closer to 1 indicates that a more precise data is in the cluster it occupies. The negative value of $S C$ indicates that data is not in the right cluster. When the value equals zero, it indicates that the data is on the border between two clusters.

\subsection{D Construction Model}

After obtaining the optimum number of clusters, the segmented image would be arranged to three-dimensional structures. When the volume is rendered in Matlab, a better visualization in 3D models is obtained. Based on the Matlab guidance, there are five steps to use the volume viewer app.

1) Load volume data into the volume viewer (this input data is the segmentation results in the previous assignment). 
2) View the volume data in the volume viewer.

3) Adjust view of volume data in volume viewer.

4) Refine the view with the rendering editor.

5) Save volume viewer rendering and camera configuration settings.

Each explanation could be found easily in Mathworks sites (see the reference [16]). The 3D model will help the radiologist to see the whole perspective of an MRI image in better visualization.

\subsection{Brain Tumor Volume Calculation}

The brain tumor volume calculations are done to each sagittal, coronal, and axial perspective. This study used the ellipsoidal approach since it is considered to be close to the actual tumor volume [17]. The formula of this approach is given by

$$
V=\frac{\pi}{6} \times \text { Lenght } \times \text { Width } \times \text { Height },
$$

where $V$ is the tumor volume, Length is the longest diameter of the tumor base, Width is the widest diameter of the tumor, and Height is the sum of slice thickness and spacing between slice (the information of slice thickness and spacing between slice is already on the MRI image properties of the patient).

\section{Implementation, Trial, Results and Discussions}

This study used a dataset from Dr. Soetomo and has been passed the medical consent. The dataset is from $\mathrm{T} 1 \mathrm{memp}+\mathrm{C}$ sequence i.e. sequences that has been added some contrast media (a type of injection before a brain scan is implemented) to clarify tumor images. The sequences are performed in sagittal, coronal, and axial perspective. Each perspective has 25 slices of images. Therefore, the total images in this study are $75 \mathrm{MRI}$ images in grayscale mode. The properties of the dataset are given in Table 1.

Table 1: Properties of Dataset

\begin{tabular}{lccc}
\hline Perspective & Number of slices & Size of image & format \\
\hline Sagittal & 25 & $256 \times 256$ & ${ }^{*}$.dcm \\
Coronal & 25 & $256 \times 256$ & ${ }^{*}$.dcm \\
Axial & 25 & $256 \times 256$ & ${ }^{*}$.dcm \\
\hline
\end{tabular}

Each image is segmented with GMM to enhance the tumor area as the region of interest. The results is discussed for one sample image in each perspective in the following sub-sections since it is not possible to discuss all in one paper. 


\subsection{MRI Image Segmentation using GMM}

In this section, dataset has been clustered using GMM couple with the EM algorithm for its optimization. This process is dividing the MRI data image into several clusters. The tumor area from an MRI image is a region of interest (ROI) that simplifies the forming of threedimensional figures. This accurately displays the tumor figures. The clustering result of several slices from each part would be discussed below.

\subsubsection{Segmentation of the MRI Axial Slice}

Clustering on the axial slice using GMM generates some output such as parameters on the histogram, clustering image result, and ROI position. The output generated is as follows.

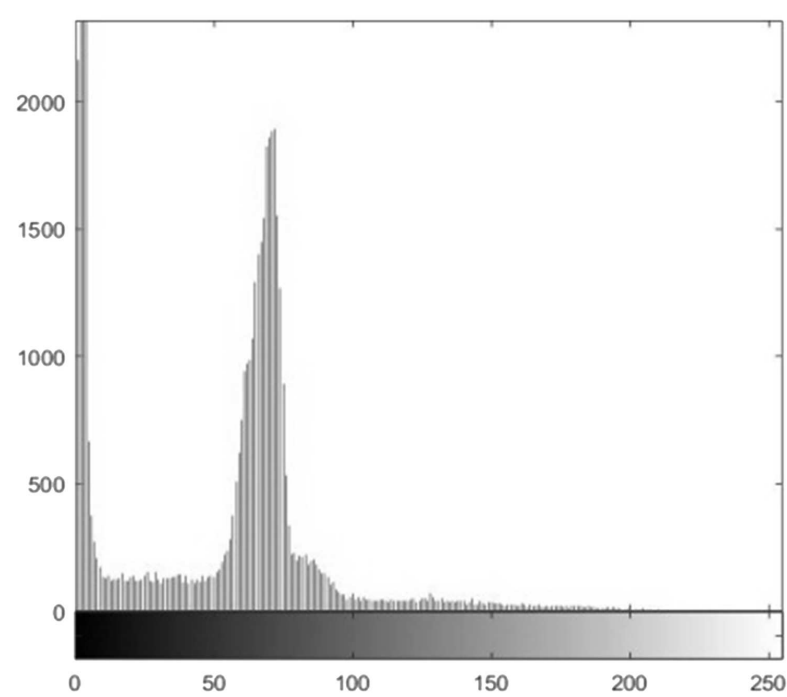

(a)

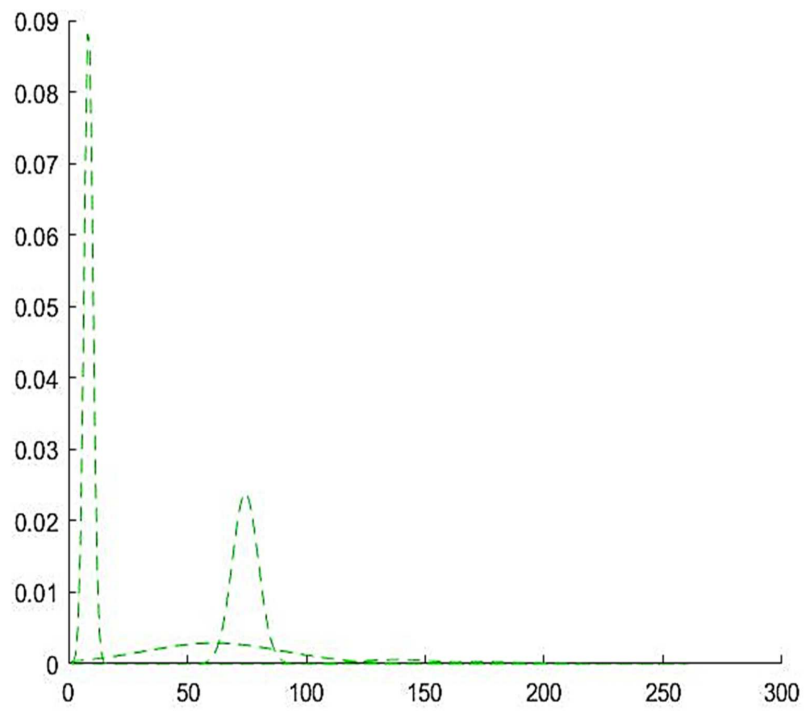

(b)

Figure 2: (a) The original $19^{\text {th }}$ Axial Slice Histogram and (b) Six Clusters of the Smoothed Histogram

Figure 2 shows the mixture pattern through the histogram formed by 6 clusters for the $19^{\text {th }}$ axial slice. This pattern is similar to the original image histogram with one parameter located to every peak. Figure 3 (a) performed the original image of the $19^{\text {th }}$ axial slice, while Figure 3 (b) is the segmentation result that contains 6 clusters.

The next step is to determine which cluster is the region of interest or ROI, with Figure 4 (a) is displaying part of Non-ROI and, in contrast, Figure 4 (b) is the ROI. The ROI is located in the $4^{\text {th }}$ cluster, since it is caught in the tumor area, while the Non-ROI is the whole clusters except the $4^{\text {th }}$ cluster members.

Figure 5 shows the clustering result evaluation using $S C$. Noise in the MRI images has different intensity values than other segments. It is, therefore, needed some additional clusters to elaborate these noises, i.e. the number of 5 to 8 clusters are then added. On the results of segmentation, ROI in 5 clusters has a large area, since it includes the Non-ROI. Therefore, 


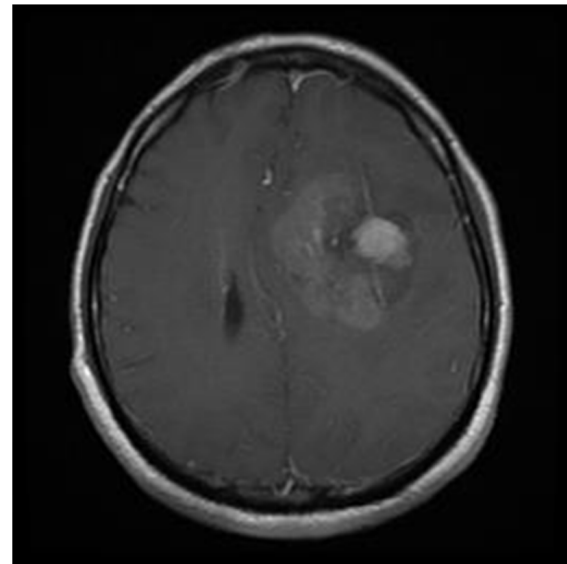

(a)

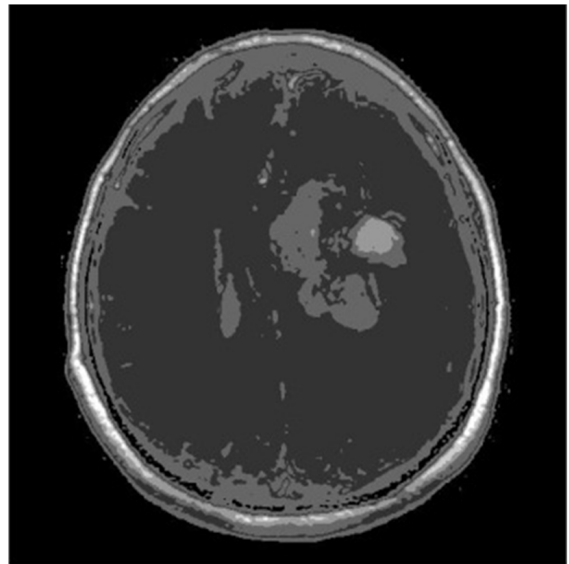

(b)

Figure 3: The $19^{\text {th }}$ Axial Slice (a) Original (b) Clustering Result

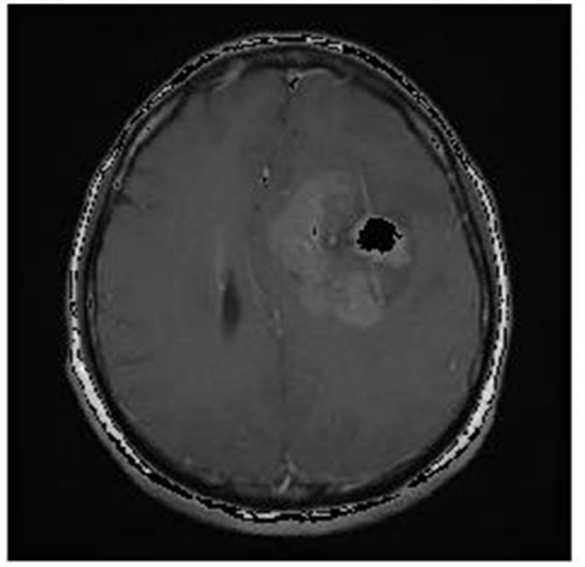

(a)

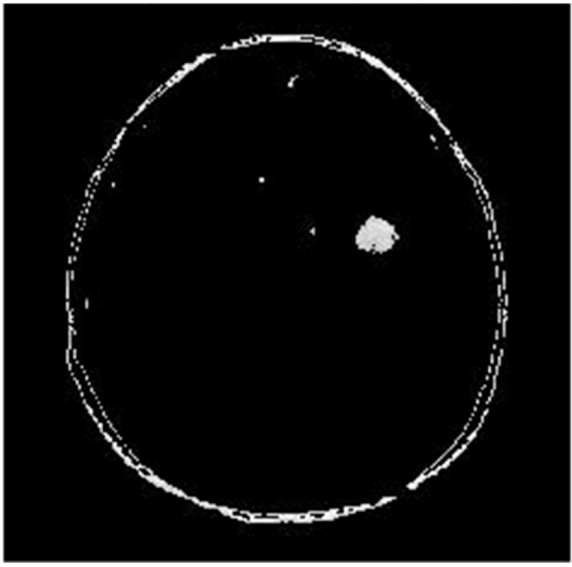

(b)

Figure 4: (a) Non-ROI and (b) ROI of the $19^{\text {th }}$ Axial Slice

even though it has the highest SC, the result of segmentation is a lack of accuracy. Medical justification gives the decision that 6 clusters are able to provide a more appropriate ROI.

\subsubsection{Segmentation of the MRI Coronal Slice}

Some outputs from the clustering process on the coronal slice include histogram, image, ROI, and Non-ROI results. The outputs are as follows:

Figure 6 shows the original image histogram and the smoothed multi-modal pattern of histogram representing a mixture model. This pattern formed 6 clusters for the $10^{\text {th }}$ coronal slice. These six peak forms can clearly elaborate the image histogram of the $10^{\text {th }}$ coronal slice. The parameters for $10^{t h}$ coronal slice are $\mu_{1}=3.553297, \mu_{2}=73.07929, \mu_{3}=68.43378$, $\mu_{4}=119.9925, \mu_{5}=168.465, \mu_{6}=185.0728, p_{1}=0.367138, p_{2}=0.20742, p_{3}=0.303886, p_{4}=0.081553$, 


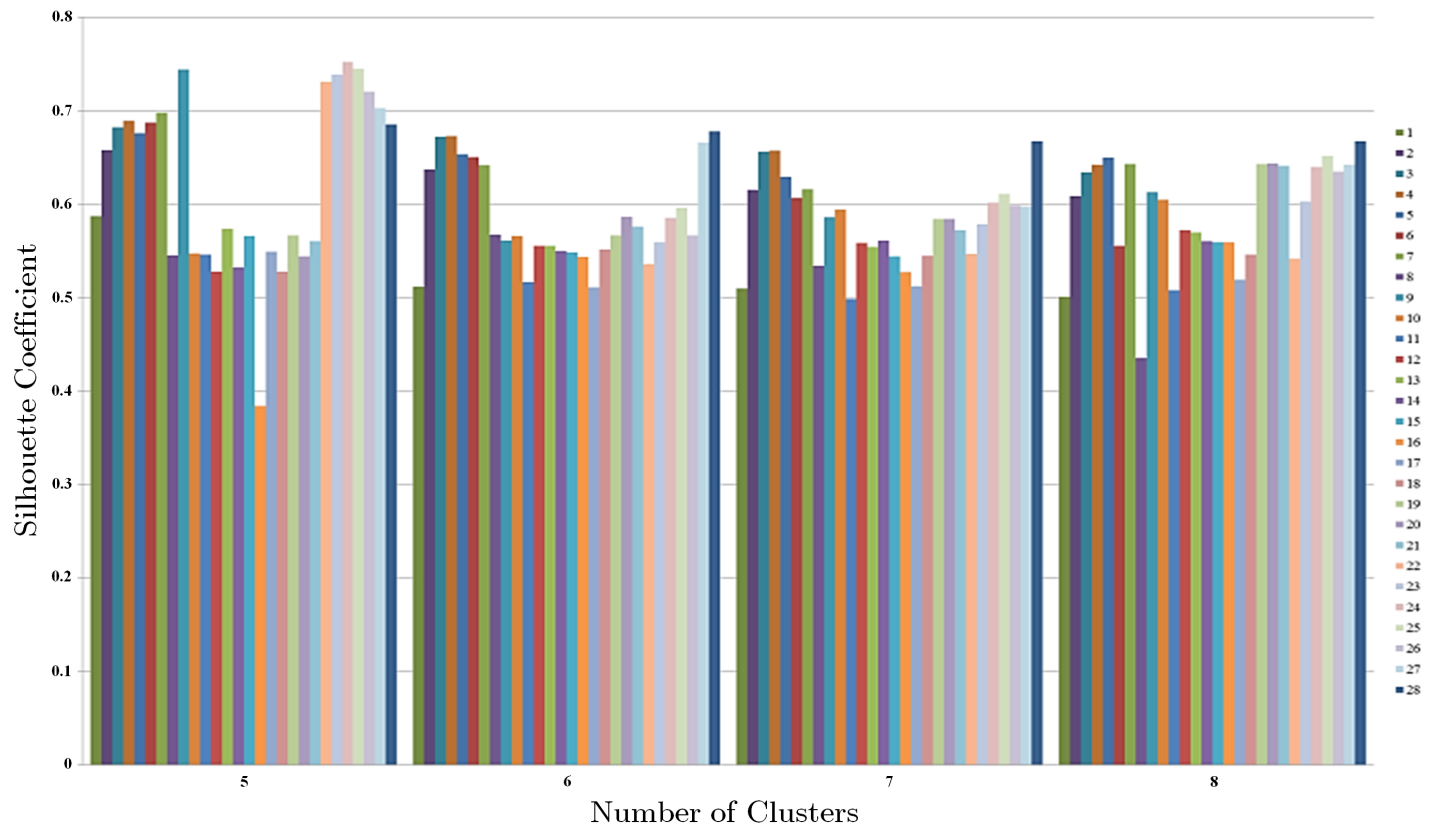

Figure 5: Comparison of $S C$ from 5 to 8 Number of Cluster in Axial Slice

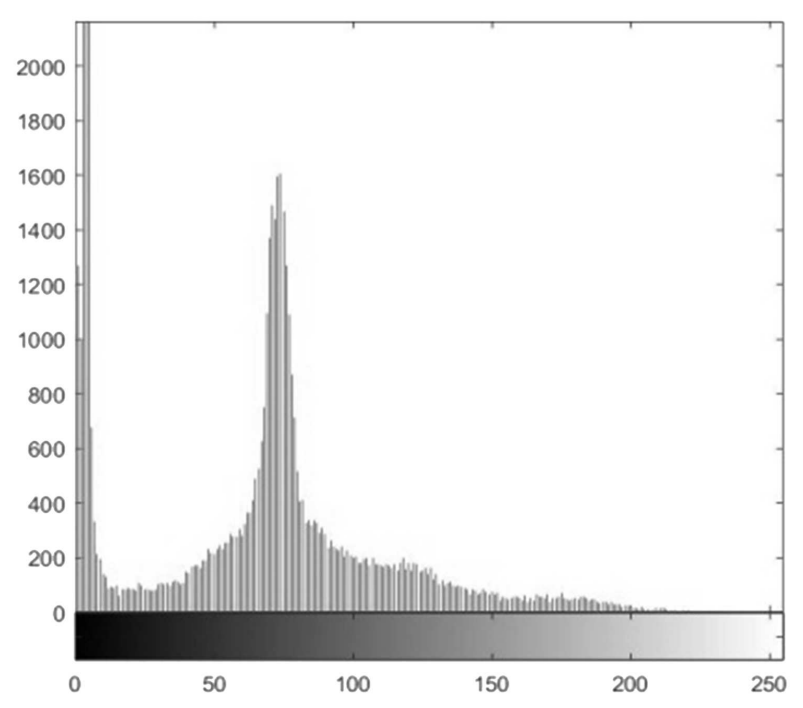

(a)

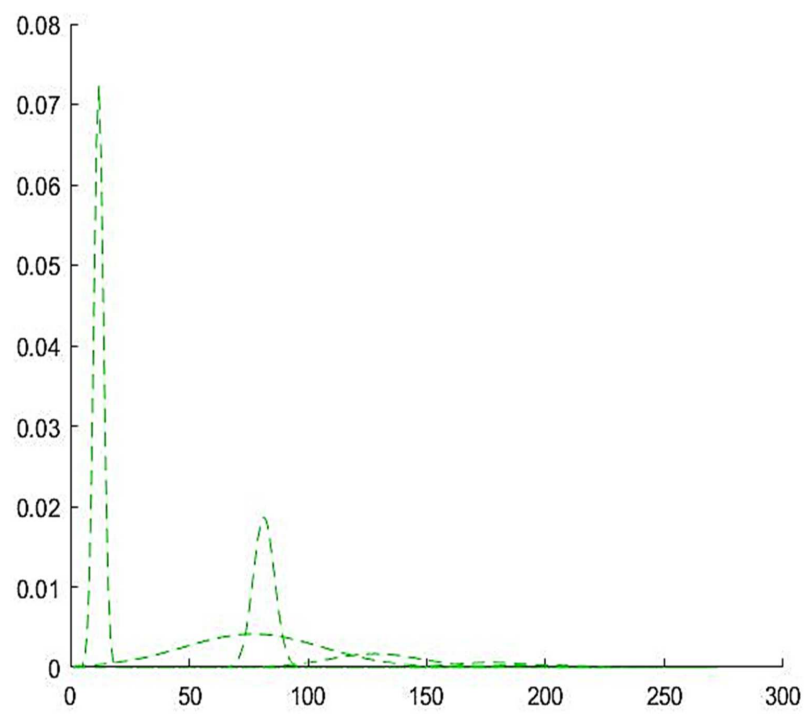

(b)

Figure 6: (a) The original $10^{\text {th }}$ Coronal Slice Histogram and (b) Six Clusters of the Smoothed Histogram

$p_{5}=0.024879, p_{6}=0.015124, \sigma_{1}^{2}=4.0967, \sigma_{2}^{2}=19.4162, \sigma_{3}^{2}=841.5028, \sigma_{4}^{2}=378.7471, \sigma_{5}^{2}=275.1054$, and $\sigma_{6}^{2}=365.8272$.

Figure 7 (a) is the original image of the $10^{\text {th }}$ coronal slice while Figure 7 (b) is an MRI data image accumulated by 6 clusters with the tumor area is in the forth. In line with the Axial 


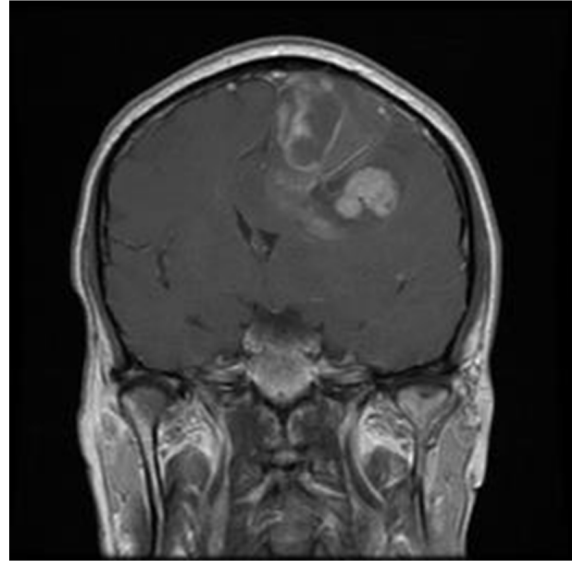

(a)

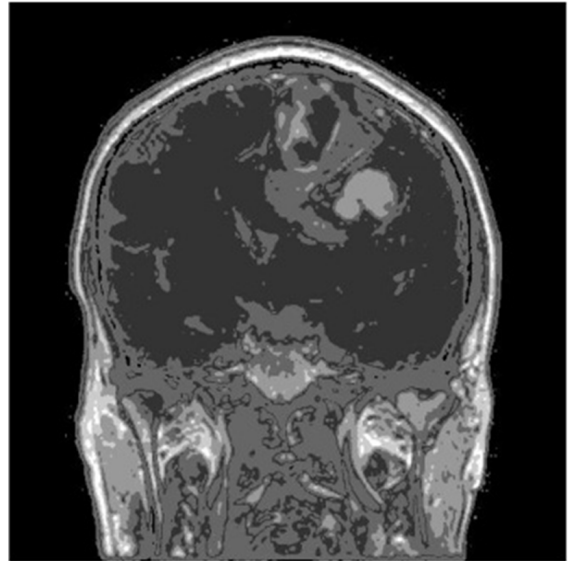

(b)

Figure 7: The $10^{\text {th }}$ Coronal Slice (a) Original (b) Clustering Result

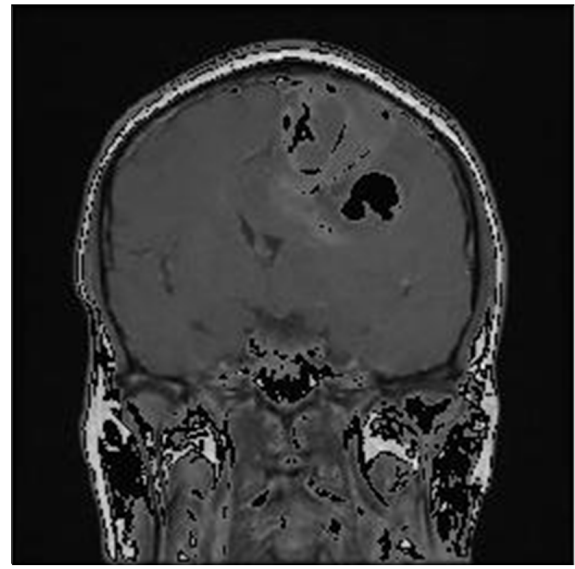

(a)

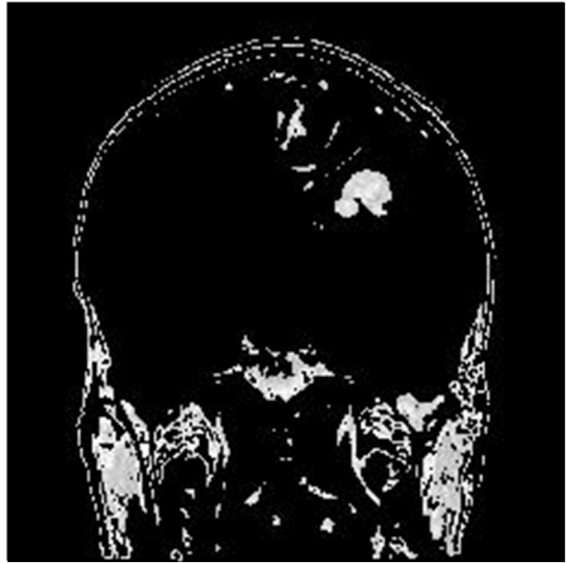

(b)

Figure 8: (a) Non-ROI and (b) ROI of the $10^{\text {th }}$ Coronal Slice

results, the selection of 6 clusters is based on medical justification. Even though 5 clusters have the largest $S C$ value, the 6 number of clusters give a better capturing the ROI. Therefore, we choose 6 as the optimum number of clusters. Figure 8 (a) shows the Non-ROI, while Figure 8 (b) is the ROI from the $10^{\text {th }}$ coronal slice. The evaluation result with $S C$ is shown in Figure 9.

\subsubsection{Segmentation of the MRI Sagittal Slice}

As two other slices before, GMM for clustering MRI sagittal slice gives histogram, image, and position of the ROI. Figure 10 shows the original image histogram and the smoothed multimodality of histogram formed by 6 clusters as a mixture model for the $16^{\text {th }}$ sagittal slice. If the 


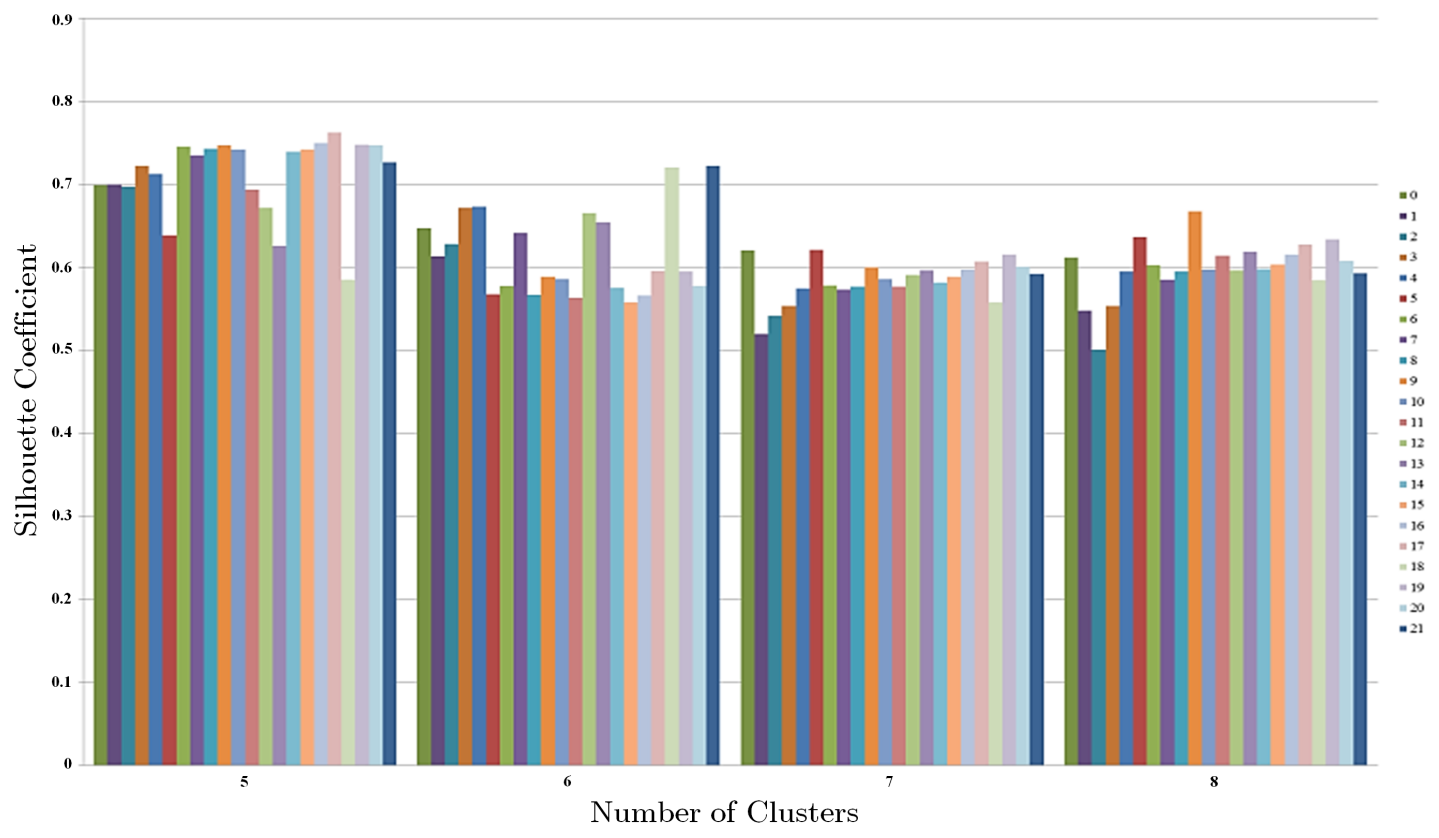

Figure 9: Comparison of $S C$ from 5 to 8 Number of Cluster in Coronal Slice

mixture model parameters are arranged, the general model is as follows.

$$
\begin{aligned}
f\left(\mathbf{x} \mid \mu_{j}, \sigma_{j}^{2}, p_{j}\right)= & \sum_{j=1}^{6} p_{j} N\left(\mathbf{x}_{i \in j} \mid \mu_{j}, \sigma_{j}^{2}\right) \\
= & p_{1} N\left(\mathbf{x}_{i \in j=1} \mid \mu_{1}, \sigma_{1}^{2}\right)+p_{2} N\left(\mathbf{x}_{i \in j=2} \mid \mu_{2}, \sigma_{2}^{2}\right)+p_{3} N\left(\mathbf{x}_{i \in j=3} \mid \mu_{3}, \sigma_{3}^{2}\right)+ \\
& p_{4} N\left(\mathbf{x}_{i \in j=4} \mid \mu_{4}, \sigma_{4}^{2}\right)+p_{5} N\left(\mathbf{x}_{i \in j=5} \mid \mu_{5}, \sigma_{5}^{2}\right)+p_{6} N\left(\mathbf{x}_{i \in j=6} \mid \mu_{6}, \sigma_{6}^{2}\right) \\
= & 0.251 N\left(\mathbf{x}_{i \in j=1} \mid 2.511,3.206\right)+0.284 N\left(\mathbf{x}_{i \in j=2} \mid 66.301,22.866\right)+ \\
& 0.363 N\left(\mathbf{x}_{i \in j=3} \mid 55.665,674.789\right)+0.066 N\left(\mathbf{x}_{i \in j=4} \mid 122.43,436.616\right)+ \\
& 0.024 N\left(\mathbf{x}_{i \in j=5} \mid 162.729,281.802\right)+0.010 N\left(\mathbf{x}_{i \in j=6} \mid 212.967,317.766\right)
\end{aligned}
$$

Figure 11 (a) is the original image of the $16^{\text {th }}$ sagittal slice while Figure 11 (b) is the MRI data image accumulated by 6 clusters after the GMM processes. The tumor area in the fourth cluster has a grayscale intensity similar to the skull region.

Figure 12 (a) shows the part of the Non-ROI segment, and Figure 12 (b) shows the ROI of the $16^{\text {th }}$ sagittal slice. The fourth cluster is the ROI because it has a tumor area in it. The $S C$ is performed by sagittal slice clustering as shown in Figure 13. It illustrates that 5 number of clusters have high $S C$ value with low slices where brain tumor is obviously seen. Nevertheless, the 6 clusters still chosen for the optimum, based on the medical justification. Since it could be able to catch the ROI appropriately.

\subsection{Visualizing the Segmented Image in a 3D Figure}

After being clustered, the next step is visualizing the three perspectives images in the threedimensional figure. This step is done for identifying the position of the tumor based on ROI which is conducted on each slice (axial, coronal, and sagittal). 


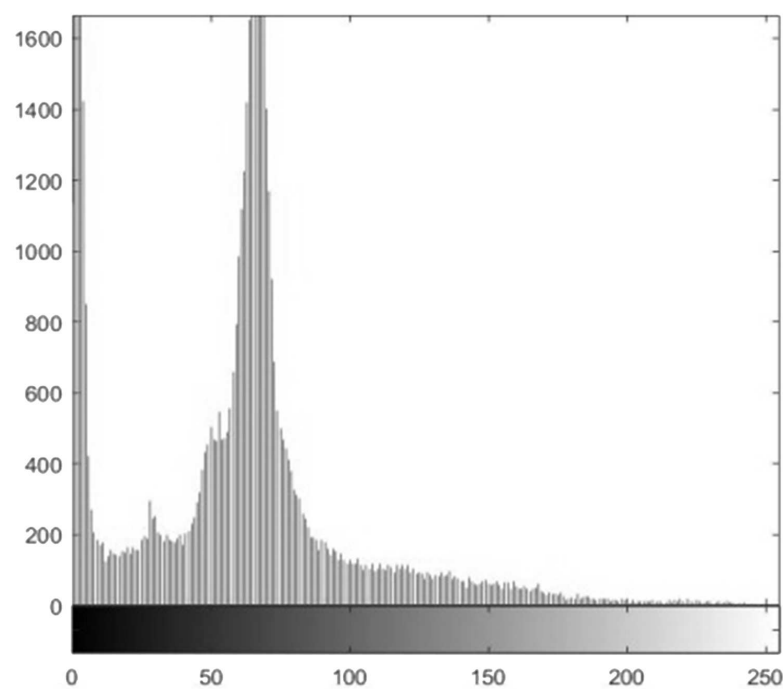

(a)

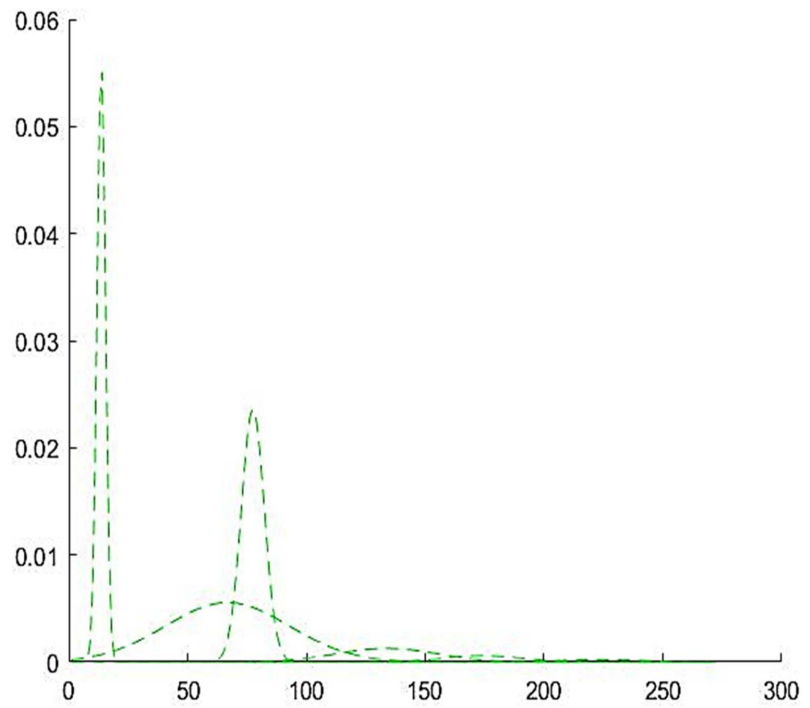

(b)

Figure 10: (a) The original $16^{\text {th }}$ Sagittal Slice Histogram and (b) Six Clusters of the Smoothed Histogram

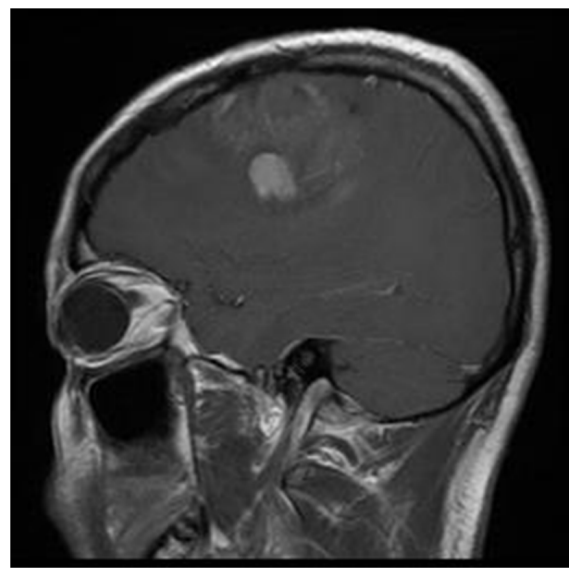

(a)

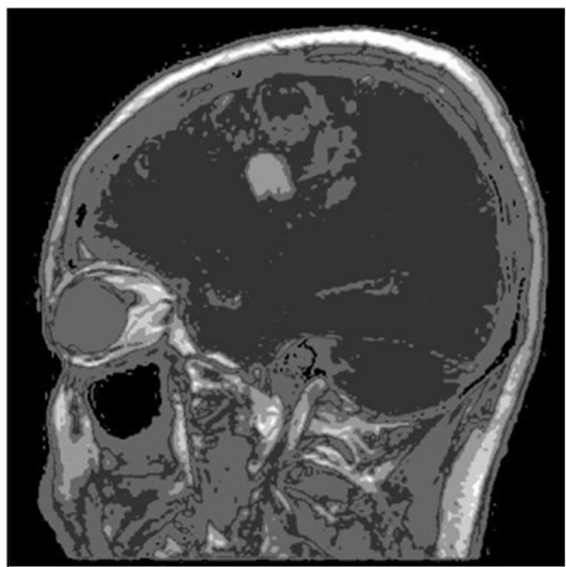

(b)

Figure 11: The $16^{\text {th }}$ Sagittal Slice (a) Original (b) Clustering Result

\subsubsection{Modelling 3D Figure of Axial Slice}

The GMM modeling to 25 number of the axial slice gives the result that is then ready to be visualized in a compact 3D figure of the axial slice. This compact 3D figure show an MRI image axial slice arranged downward according to the picture sequence which partially forms the patients head. ROI caught from the clustering result of the $19^{t h}$ axial slice where the tumor figure is obviously seen can be used to represent its part.

Figure 14 is a three-dimensional figure of the axial slice from various views though the head 


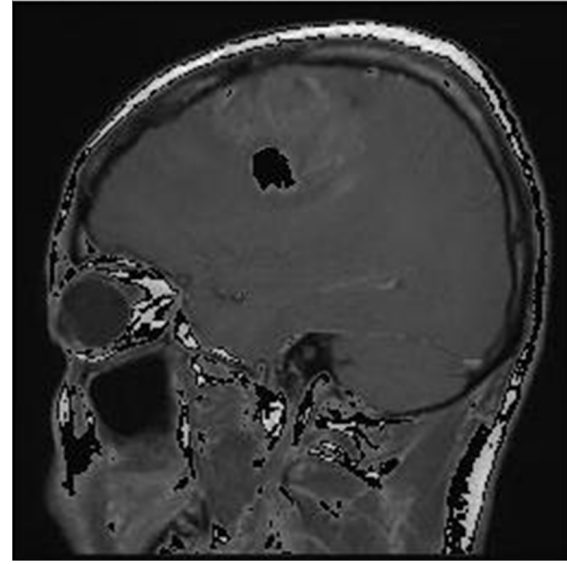

(a)

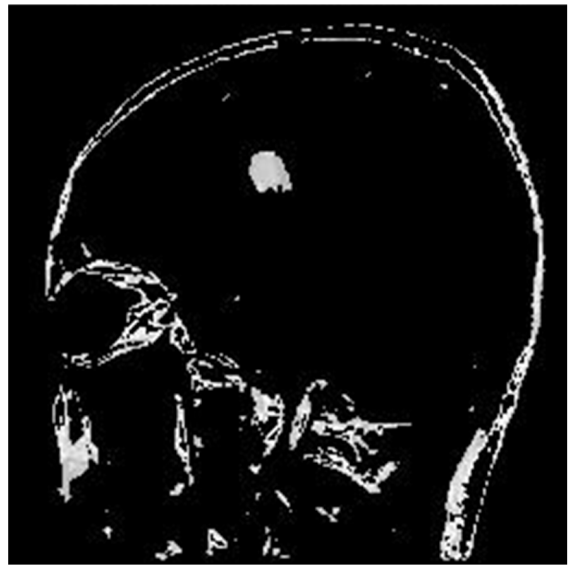

(b)

Figure 12: (a) Non-ROI and (b) ROI of $16^{\text {th }}$ Sagittal Slice

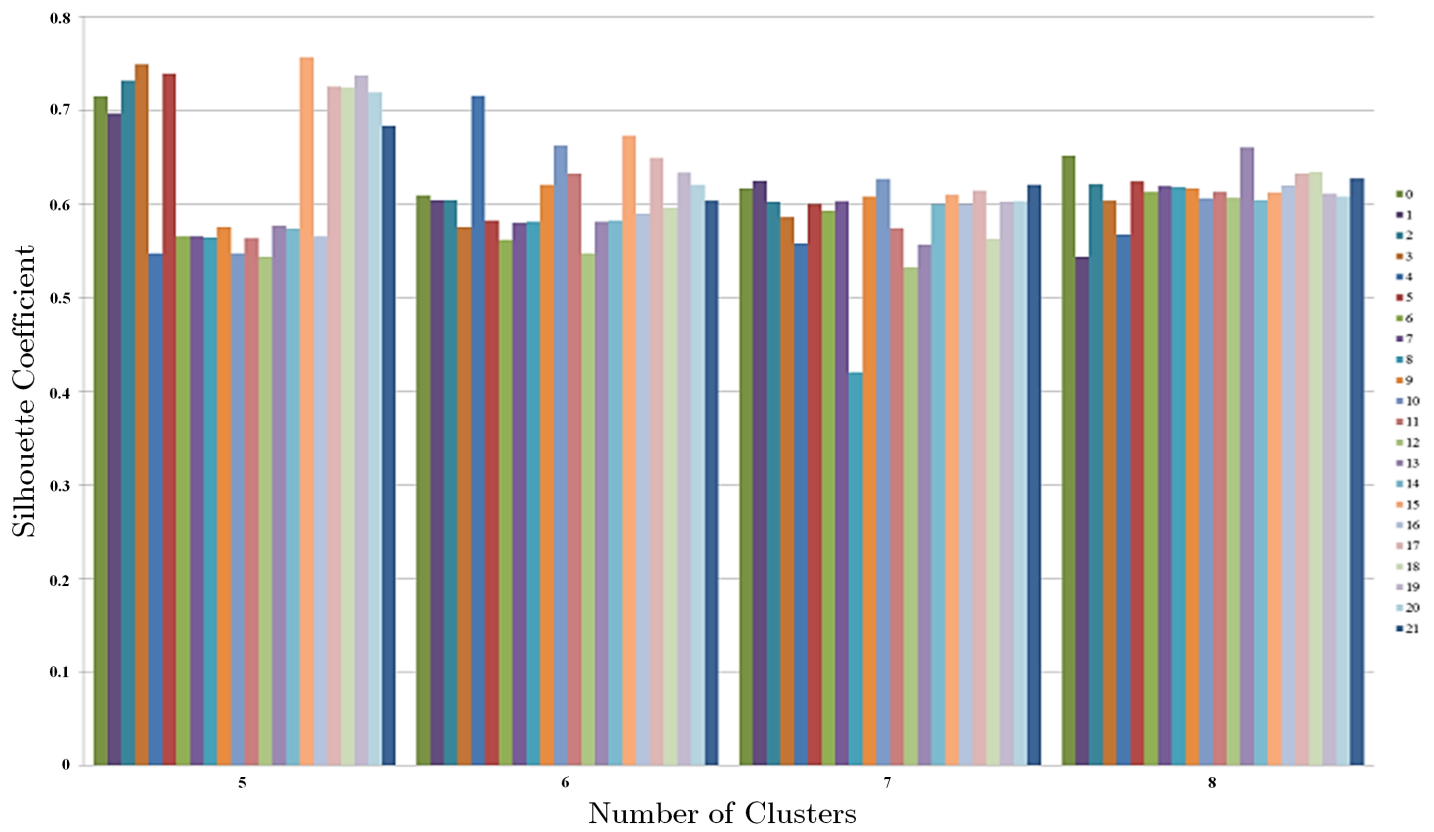

Figure 13: Comparison of $S C$ from 5 to 8 Number of Cluster in Sagittal Slice

section is only partially represented owing to the fact that the MRI data image is focused on the brain part. Figure 14 (a) displays a 3D figure from the left side of the patient's head, Figure 14 (b) shows from the bottom point of view, and Figure 14 (c) displays from the top point of view.

In order to display the tumor inside the brain, the opacity is adjusted by using the feature located on the right. The result is shown in Figure 15. 


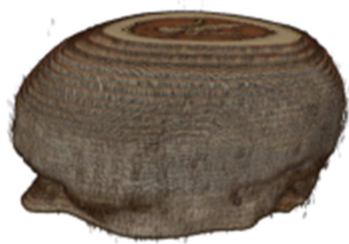

(a)

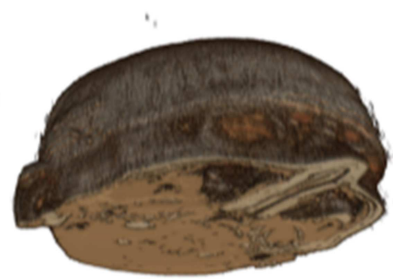

(b)

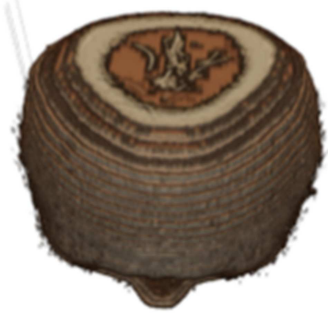

(c)

Figure 14: 3D Figures of MRI Axial Slice from the (a) left, (b) bottom, and (c) top viewpoints

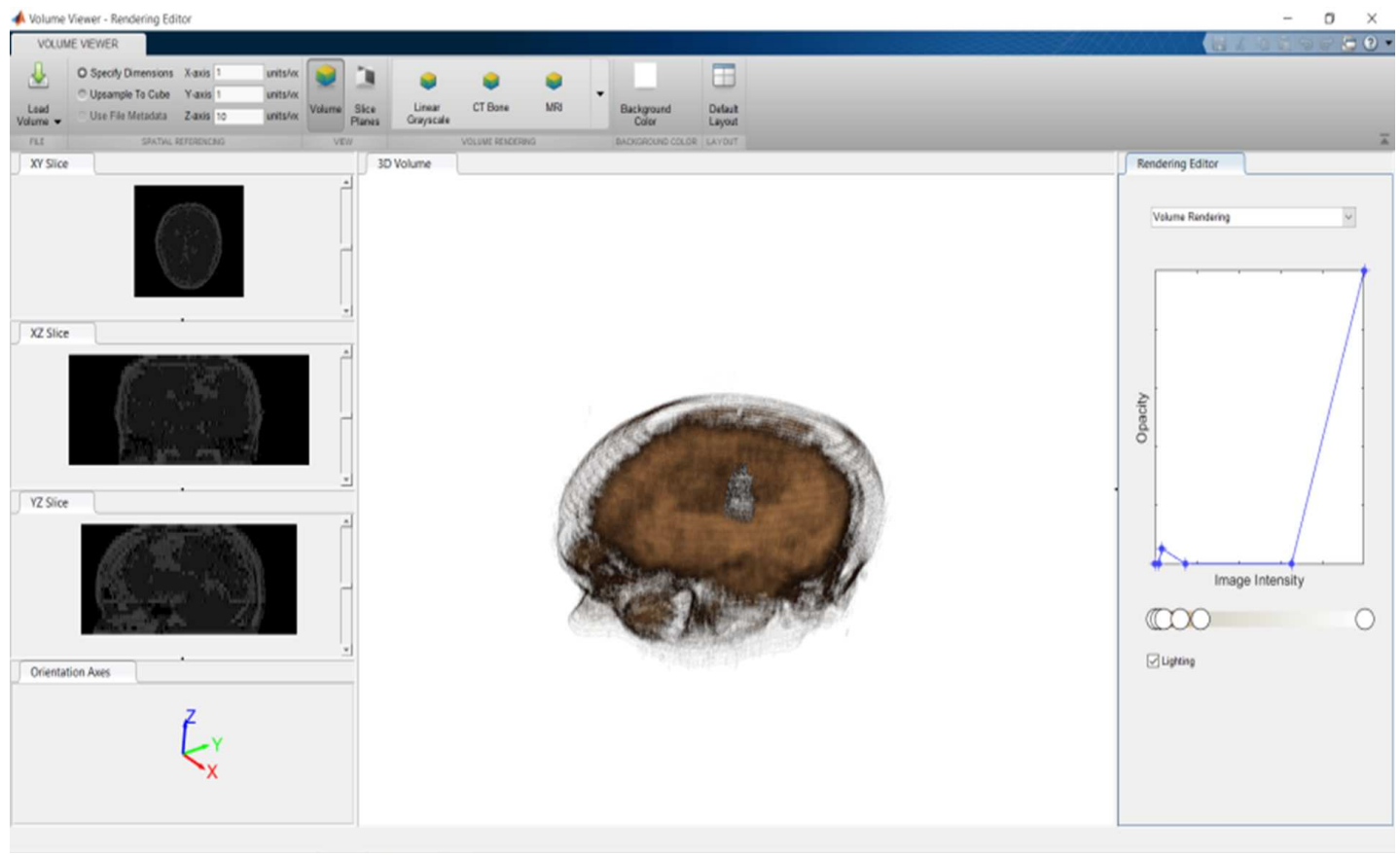

Figure 15: Tumor Position in 3D Figures of MRI Axial Slice

\subsubsection{Modelling 3D Figure of Coronal Slice}

Coronal slice is a representation of the head from front to back. Every coronal slice is arranged backward according to the sequence. The clustering result of the $10^{\text {th }}$ slice is used to analyze brain tumor shape because the ROI clearly describes it.

The GMM modeling to 25 number of the coronal slice gives the result that is then ready to be visualized in a compact 3D figure of the coronal slice. This compact 3D figure of the coronal slice are shown in Figure 16. It displays part of the patient's head with clustering results used to determine the color. Figure 16 (a) displays a 3D figure from the left side of the patient's head, Figure 16 (b) shows from the right side, and Figure 16 (c) displays from the front side. In Figure 18 brain tumor is not visible because it is inside. In Figure 17, we try to display the tumor area inside the brain by configuring the opacity of the volume viewer editor. The results, somehow, are less visible unlike in 3D images of axial slices. 


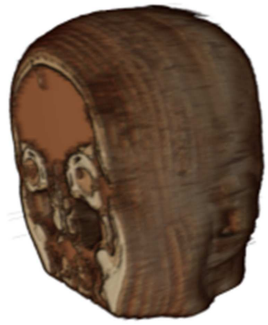

(a)

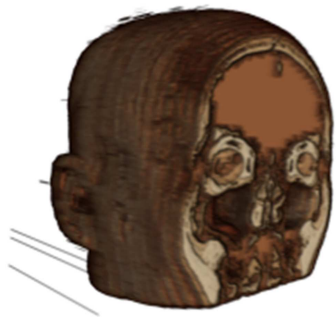

(b)

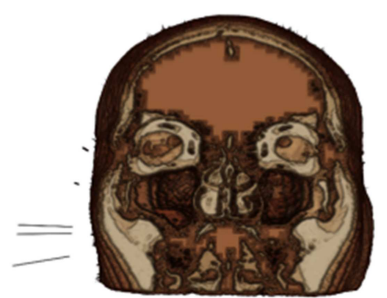

(c)

Figure 16: 3D Figures of MRI Coronal Slice from (a) left, (b) right, and (c) front viewpoints

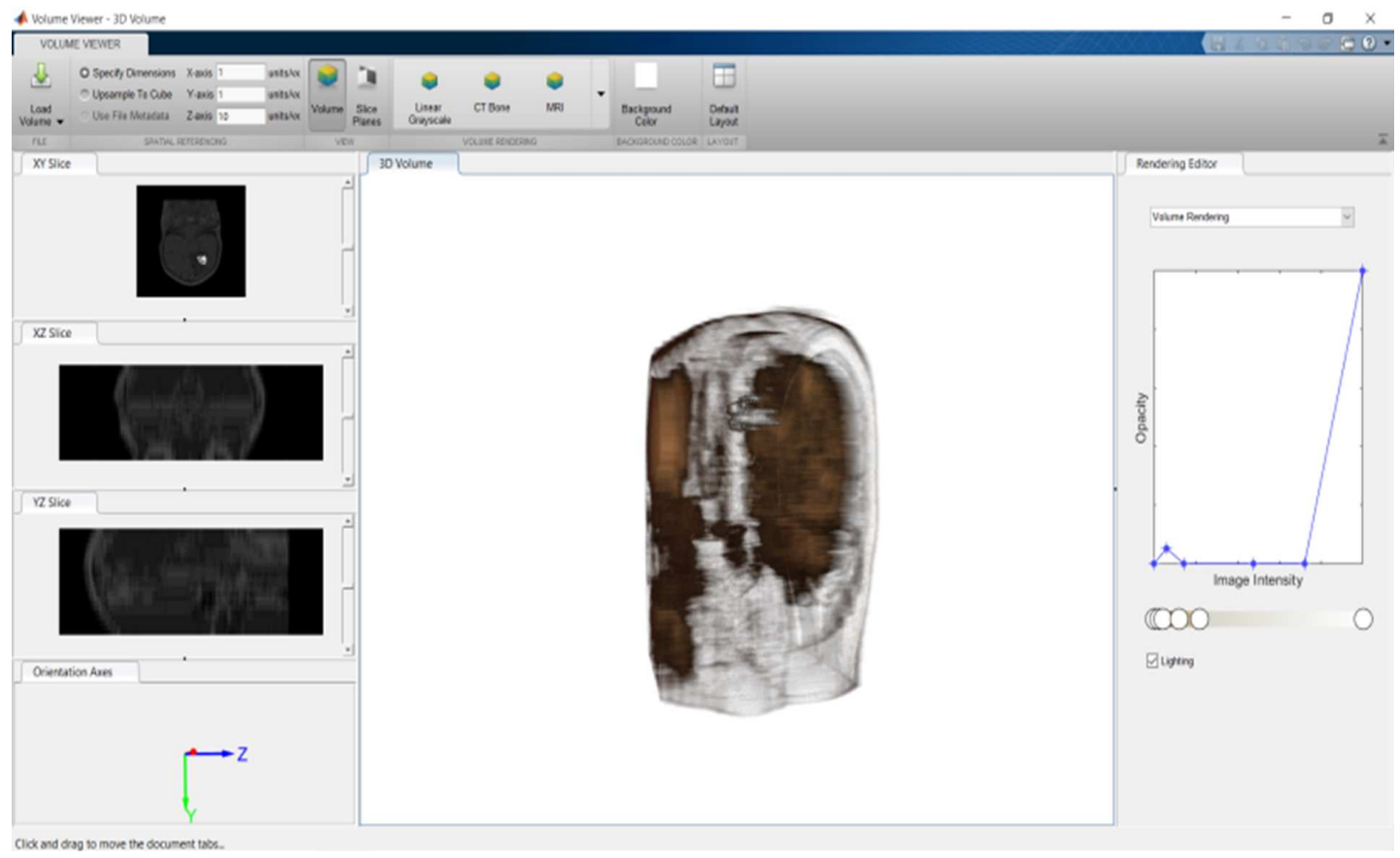

Figure 17: Tumor Position in 3D Figures of MRI Coronal Slice

\subsubsection{Modelling 3D Figure of Sagittal Slice}

Three-dimensional figures from sagittal slice show an MRI image horizontally arranged according to the sequence that forms part of the patients head. The ROI that represents the tumor figure is taken from the $16^{\text {th }}$ slice.

The GMM modeling to 25 number of the sagittal slice demonstrates the segmentation result that is then ready to be visualized in a compact 3D figure of the sagittal slice. This compact 3D figure is shown in Figure 18. Figure 18 (a) displays a 3D figure from the left side of the patient's head. Both Figure 18 (b) and Figure 18 (c) are the display from the right point of view. The tumor location is described in Figure 19. The head section formed is not complete, however, it is located inside the brain but would be made transparent. Based on modeling three-dimensional figures of the axial, coronal, and sagittal slice, it is known that the tumor is located on the left side of the patients head. 


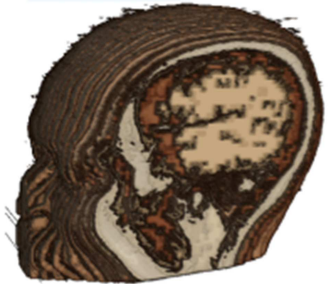

(a)

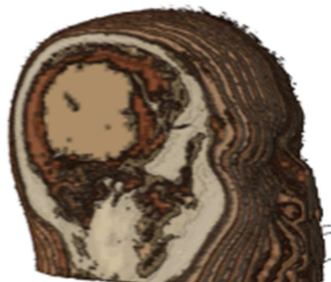

(b)

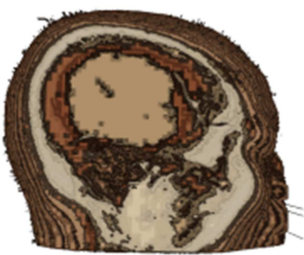

(c)

Figure 18: 3D Figures of MRI Sagittal Slice from (a) left, and (b, c) right viewpoints

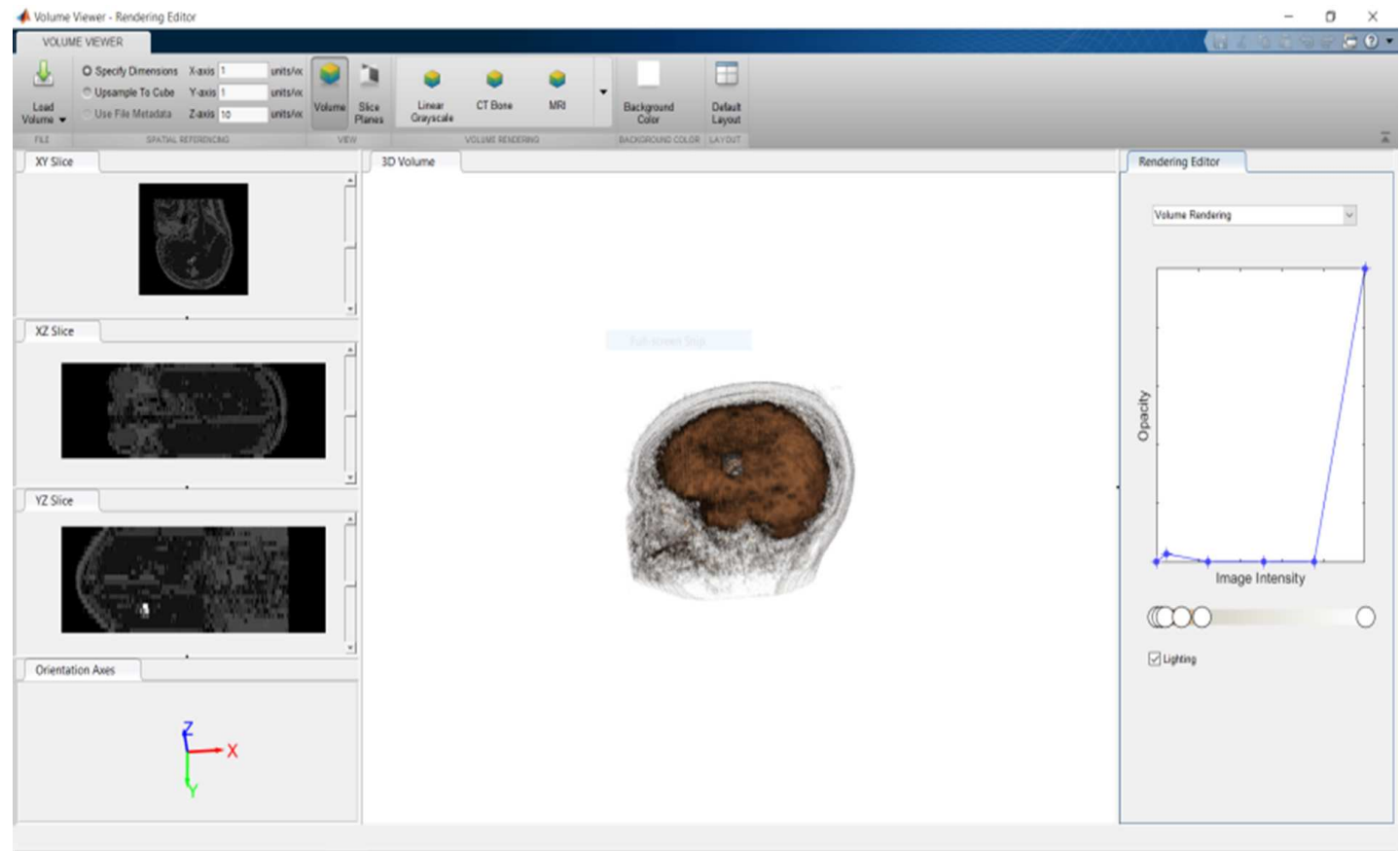

Figure 19: Tumor Position in 3D Figures of MRI Sagittal Slice

\subsection{Calculating the Tumor Volume}

Table 2 shows the resume of the tumor volume calculation for each perspective with the ellipsoidal approach. The Length and Width of each perspective are measured from the results of ROI in image segmentation. Table 2 shows the calculation of tumor volume in each perspective having similar results about $749 \mathrm{~mm}^{3}$.

\section{Conclusion}

In terms of prediction of the number of distinguishing image clusters, the GMM couple with EM algorithms approach provides precise and powerful image segmentation. This approach has been applied to make segmentation of MRI image in three perspective input slices. This study had succeeded to construct the compact three-dimensional image from two-dimensional MRI sequences by combining three perspective input slices in a compact manner. The results can show a 3D model giving fairly good results. Moreover, from the analysis, we can see that the 
Table 2: Resume of Tumor Volume Calculation

\begin{tabular}{lcccc}
\hline Perspective & Legth $(\mathrm{mm})$ & Width $(\mathrm{mm})$ & Height $(\mathrm{mm})$ & Volume $\left(\mathrm{mm}^{3}\right)$ \\
\hline Sagittal & 11.39 & 10.99 & 11.39 & 746.524 \\
Coronal & 11.28 & 11.64 & 10.88 & 747.979 \\
Axial & 11.73 & 10.76 & 11.39 & 752.719 \\
\hline Average & \multicolumn{5}{c}{} & 749.074 \\
\hline
\end{tabular}

tumor is located on the left side of the patients head and have an estimated volume of about $749 \mathrm{~mm}^{3}$.

\section{Acknowledgments}

The Authors are grateful to the Directorate for Research and Community Service (DRPM) Ministry of Research, Technology, and Higher Education Indonesia which supported this research under PT research grant no. 1311/PKS/ITS/2020.

\section{References}

[1] Globocan. Indonesia cancer fact sheets. url: http://gco.iarc.fr/today/data/factsheets/ populations/360-indonesia-fact-sheets.pdf. 2109.

[2] Rofiky, A., Rahardjo, P. and Soeharmanto, D. Comparison of number of diffusion gradient direction in brain imaging diffusion tensor; case study of tumor brain. Journal of Vocational Health Studies. 2017. 1: 15-17. DOI: 10.20473/jvhs.V1.I1.2017.15-17.

[3] Martínez-Usó, A., Pla, F., and García-Sevilla, P. Unsupervised image segmentation using a hierarchical clustering selection process. In Structural, Syntactic, and Statistical Pattern Recognition, pages 799-807. Springer Berlin Heidelberg. 2006. DOI: 10.1007/11815921_88.

[4] Pestunov, I. A., Rylov, S. A., and Berikov, V. B. Hierarchical clustering algorithms for segmentation of multispectral images. Optoelectronics, Instrumentation and Data Processing. 2015. 51: 329338. DOI: 10.3103/S8756699015040020.

[5] Rohith, J., Ramesh, H. Colour based segmentation of a landsat image using k-means clustering algorithm. Journal of Image Processing \&3 Pattern Recognition Progress. 2017. 4(3): 3138 .

[6] Muruganandham, S.K., Sobya, D., Nallusamy, S., Mandal, D.K. and Chakraborty, P.S. Study on leaf segmentation using k-means and k-medoid clustering algorithm for identification of disease. Indian Journal of Public Health Research 83 Development. 2018. 9(5): 289-293.

[7] Huang, H., Meng, F., Zhou, S., Jiang, F. and Manogaran, G. Brain image segmentation based on FCM clustering algorithm and rough set. IEEE Access, pages 12386-12396. 2019.

[8] Lee, T.H., Fauzi, M.F.A., Komiya, R. and Haw, S.C. Unsupervised abnormalities extraction and brain segmentation. In 2008 3rd International Conference on Intelligent System and Knowledge Engineering, 1: 1185-1190. IEEE. 2008. DOI: 10.1109/ISKE.2008.4731110. 
[9] Oh, M. S. and Raftery, A. E. Model-based clustering with dissimilarities: a Bayesian approach. Journal of Computational and Graphical Statistics. 2007. 16(3): 559-585.

[10] Shi, H. S. Model-based Clustering. Ontario: University of Waterloo. 2005.

[11] Greve, B., Pigeot, I., Huybrechts, I., Pala, V., and Brnhorst, C. A comparison of heuristic and model-based clustering methods for dietary pattern analysis. Public Health Nutrition. 2015. 1-10.

[12] Kumar, N. S., Satoor, S. and Buck, I. Fast parallel expectation maximization for gaussian mixture model on GPUs using CUDA. 11th IEEE International Conference on High Performance Computing and Communications. 2009. 103-105. DOI: 10.1109/HPCC.2009.45.

[13] Pravitasari, A. A., Qanita, S. F., Iriawan, N., Fithriasari, K., Irhamah, Purnami, S. W., and Ferriastuti, W. MRI-based brain tumor segmentation using gaussian and hybrid gaussian mixture model-spatially variant finite mixture model with expectationmaximization algorithm. Malaysian Journal of Mathematical Sciences. 2020. 14(1): 77-93.

[14] Nguyen, T. Gaussian Mixture Model Based Spatial Information Concept for Image Segmentation. Electronic Theses and Dissertation 438, https://scholar.uwindsor.ca/etd/438. 2011.

[15] Thinsungnoena, T., Kaoungkub, N., Durongdumronchaib, P., Kerdprasopb, K., and Kerdprasopb, N. The clustering validity with silhouette and sum of squared errors. Learning 2015. 3:7.

[16] Matlab. Help, section: Explore 3-D Volumetric Data with Volume Viewer App. url: https://www.mathworks.com/help/images/explore-3-d-volumetric-data-with-volumeviewer-app.html\#responsive_offcanvas. Access in 2020.

[17] Richtig, E., Langmann, G., Mllner, K., Richtig, G., and Smolle, J. Calculated tumour volume as a prognostic parameter for survival in choroidal melanomas. Eye. 2004. 18(6): 619-623. 